\title{
Agôn
}

Revue des arts de la scène

$4 \mid 2011$

L'objet

\section{Détournement et invention de l'objet scénique dans le théâtre de Dario Fo}

\section{Dominique Budor}

\section{(2) OpenEdition \\ Journals}

Édition électronique

URL : http://journals.openedition.org/agon/2056

DOI : 10.4000/agon.2056

ISSN : 1961-8581

Éditeur

Association Agôn

Référence électronique

Dominique Budor, «Détournement et invention de l'objet scénique dans le théâtre de Dario Fo », Agôn [En ligne], 4 | 2011, mis en ligne le 25 janvier 2012, consulté le 17 avril 2020. URL : http:// journals.openedition.org/agon/2056 ; DOI : https://doi.org/10.4000/agon.2056 


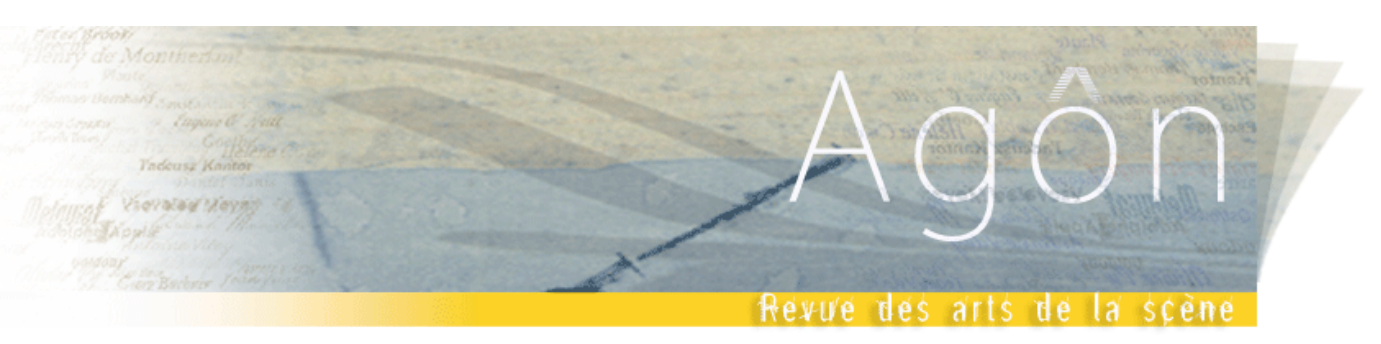

\title{
Détournement et invention de l'objet scénique dans le théâtre de Dario Fo
}

Dominique Budor

\begin{abstract}
L'article examine le traitement de l'objet (entendu comme élément non humain d'occupation de l'espace scénique) dans le "théâtre d'Acteur" qu'écrit et pratique Dario Fo. Au-delà de certains usages correspondant à la revisitation et au dévoilement de la tradition comique du travestissement et $\mathrm{du}$ quiproquo, l'objet assume une fonction structurante, qu'il s'agisse de l'objet matériellement présent sur scène et manipulé par l'acteur ou bien de l'objet image mentale créée par le mime pour le spectateur. La puissance d'une "action" scénique (où l'acteur lie verbe, geste et objet) ainsi qu'une scénographie qui l'hybridise et lui confère des fonctions pragmatiques inédites arrachent l'objet à sa signification iconique, à son statut théâtral et même parfois à son essence. La perturbation des paradigmes perceptifs suscite en le spectateur des codes autres de déchiffrement; et c'est dans ce décalage généralisé que se développe la conscience critique du public par laquelle le travail de Fo atteint son efficacité politique.
\end{abstract}

\section{L'acteur et l'objet}

$\mathrm{Si}$, selon la voie sémiologique ouverte par Anne Ubersfeld, on entend par objet scénique tout élément non humain d'occupation de l'espace de la scène ${ }^{1}$, on peut, a priori et dans une approche superficielle, douter de l'importance que pourrait revêtir cette « chose » dans le théâtre de Dario Fo. De fait, des modalités spécifiques de réception - ce sont assurément des distorsions, mais elles sont efficaces - ont, vu l'exceptionnelle puissance de Fo en scène, induit au niveau du public et d'une partie de la critique (les détracteurs de l'auteur, en général), une focalisation sur la prestation actorielle, au détriment de la compréhension syncrétique des diverses composantes de la théâtralité. La réduction de l'œuvre théâtrale de Fo à l'apparence et à la présentification de cette œuvre qu'effectue le comédien au moment de la représentation n'est d'ailleurs pas innocente dans l'Italie du XXe siècle : elle sert à disqualifier l'œuvre au profit de ses interprètes, et donc à interdire aux créations de

Anne, Ubersfeld, «L'objet théâtral », in Actualité des arts plastiques, n 40, Paris, CNDP, 1978 ; «L'objet », in Les termes clés de l'analyse du théâtre, Paris, Seuil, 1996, p. 61-63. 
Fo toute existence au-delà de l'événement théâtral; la perturbation politique produite s'inscrit alors dans l'éphémère de l'ici-maintenant. L'opération est aussi intra-théâtrale, face à une œuvre qui résiste à toute catégorisation générique stable et rassurante : les questions que ce théâtre "autre" pose à la tradition italienne et à l'art théâtral en général se voient escamotées par le renvoi aux caractéristiques individuelles du jeu. C'est sur ces bases que nombre de critiques et universitaires italiens, et non des moindres, se sont dits scandalisés par l'attribution à Dario Fo en 1997 du prix Nobel de Littérature ${ }^{2}$, prix qui reconnaissait, outre la force de la performance individuelle et la vigueur de la contestation, la complexité textuelle et scénique du travail d'un représentant du théâtre total s'il en est - comme le fut autrefois Molière, dont Fo recueille si amplement les enseignements. Il est vrai que Fo lui-même, lorsqu'il défend ses textes en alléguant l'impressionnant succès de son théâtre joué par d'autres que lui, dans d'innombrables pays et langues, doit consentir à cette polarisation sur sa personne : soit à ce que le critique Luigi Allegri définit comme un effet de multiplication, Fo joué par Fo produisant « une opération dans laquelle $1+1$ fait $3^{3} »$. Car cet auteur-acteur-scénographe-metteur en scène-membre et directeur de troupe ${ }^{4}$, dont les textes échappent à la fixité de l'écrit et ne sont publiés qu'après l'épreuve de la scène $e^{5}$, ne cesse de proclamer l'absolue primauté de l'acteur sur tout autre élément théâtral. À cette promotion d'un "théâtre d'Acteur" correspond en outre l'effet de constitution en protagoniste de Dario Fo lui-même : et ce, d'autant plus facilement que la construction même des dialogues et du jeu dans les pièces confère progressivement au personnage incarné par Dario Fo la valeur d'émetteur privilégié de la force comique ou satirique et la fonction d'adresse directe au public (voir le rôle de l'anti-prologue et du prologue). Le spectateur distingue alors sur scène «l'acteur de conduite» (Fo lui-même), «l'acteur de soutien» (Franca Rame) et «les bons équipiers», comme les nomme Fo en évoquant la structuration d'une course cycliste.

Qui plus est, cette centralité de l'acteur est renforcée par le contraste qu'elle constitue avec les habitudes mentales et visuelles du spectateur moyen avant 1968 puis dans les années soixante-dix et, donc, avec le système d'attentes que tout spectacle théâtral convoque. Car, à une époque qui, pour ne citer que quelques

2 Cf. La Repubblica, 10 octobre 1997, section Culture, p. 15.

3 Dario, Fo, Dialogo provocatorio sul comico, il tragico, la follia e la ragione con Luigi Allegri, Bari, Laterza, 1990, p. 87-88. [Toutes les traductions ont été faites par l'auteur de l'article]

La variation des fonctions assumées par Fo (directeur de troupe au temps de la Compagnie Fo-Rame, organisateur du collectif Associazione Nuova Scena ou membre de la coopérative théâtrale La Comune...) n'implique pas seulement des tâches logistiques et financières différentes. Elle engendre des équilibres différents dans la conception des personnages qu'incarneront les acteurs, dans la structuration actantielle des pièces et dans les relations proxémiques sur scène.

5 Les premiers états de rédaction sont corrigés en des versions modifiées au fil d'un long travail avec Franca Rame (il y a, en des modalités variables, une sorte d'écriture à quatre mains) et par lecture devant la famille-tribu. D'autres ajustements interviennent au fil des répétitions et, enfin, pendant et après les représentations, selon l'expérience des acteurs et la réaction des différents publics (substitutions, ajouts, suppressions, voire - rarement toutefois -abandon d'une pièce). C'est ce texte en devenir que Franca Rame édite en des fixations provisoires. La déclaration de Dario Fo dans son discours de réception du Prix Nobel sur le partage avec sa «compagne de vie et d'art » de la médaille reçue, constitue ainsi, au-delà du légitime hommage, la salutaire reconnaissance d'un processus de création très particulier. 
exemples de la seconde génération de l'avant-garde théâtrale italienne, voit s'affirmer la grande machinerie figurative de Mario Ricci (Moby Dick en 1971), les jeux visuels de Memè Perlini (Pirandello chi ? en 1973) ou la promotion de l'objet au rôle de protagoniste du spectacle par le Club-Teatro de Claudio Remondi et Riccardo Caporossi ${ }^{6}$, le spectateur, dans le théâtre de Dario Fo, peut être placé en présence du seul acteur. Certes, Fo écrit, monte et joue des pièces avec décor, costumes et accessoires mais il est aussi, et peut-être d'abord, le «jongleur » qui, simplement vêtu d'un pantalon et d'un pull-over de ville, dit ses monologues sur un plateau nu; et l'on sait que Mistero buffo (Mystère bouffe ${ }^{7}$ ), proféré dans ces conditions, a été et demeure son texte le plus célèbre :

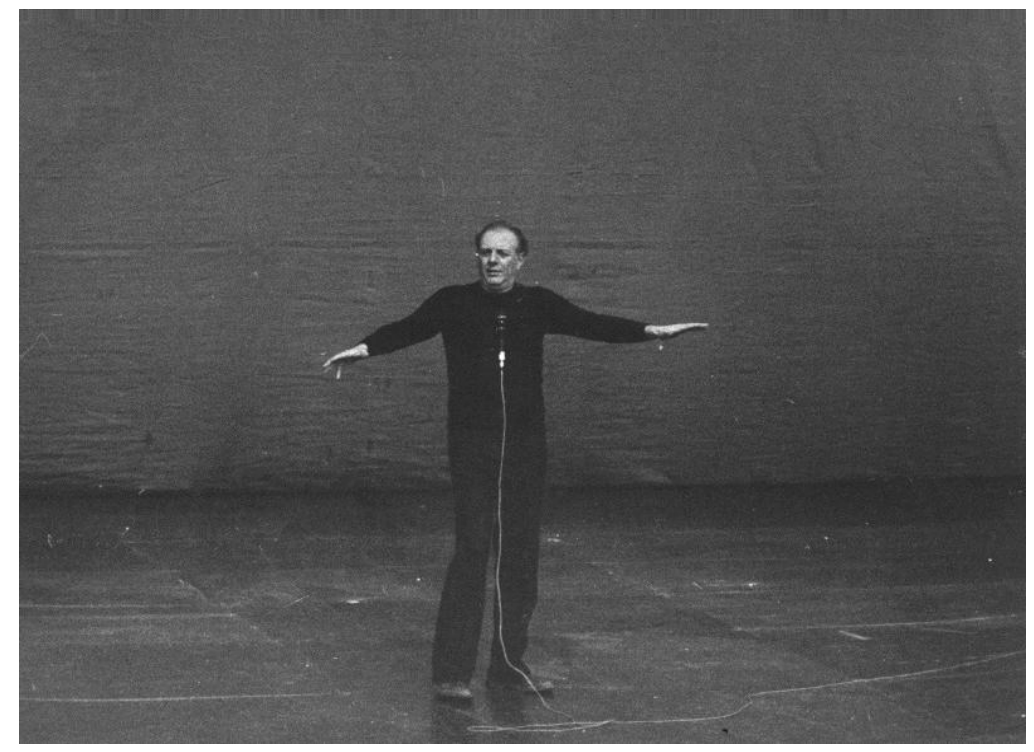

\section{Mistero buffo, ( ) /archivioforame/MIBU/1970/FOTO/006414/006414-164m}

Dans cette dramaturgie, non assimilable à toute autre écriture verbale et non verbale du $\mathrm{XX}^{\mathrm{e}}$ siècle, l'acteur est le véritable point d'engendrement et l'axe porteur du spectacle, ce qui confère une existence spécifique à l'objet: l'objet peut être matériellement absent du spectacle et, néanmoins, sémantiquement signifiant. Car l'«action théâtrale », telle que l'entend Fo, implique une totale solidarité de la

\footnotetext{
$6 \quad$ Voir, pour ce détournement de la fonction usuelle de l'objet et, sur la scène, le renversement du rapport dominé-dominant qui unit cet objet à l'homme, les déclarations des auteurs citées in Franco, Quadri, L'avanguardia teatrale in Italia (II. Materiali 1960-1976), Turin, Einaudi, 1977, p. 541-558.

Mistero buffo, créé en Italie en 1969, a été souvent repris en France dans des contextes divers : le texte a été joué devant les ouvriers de Lip, au Festival d'Avignon en 1973 et il figure désormais au répertoire de la Comédie française. Il a été traduit en 1984 par Ginette Herry sous le titre Mystère bouffe. Jonglerie populaire puis mis en scène par Muriel Mayette sous celui de Mystère bouffe et fabulages en 2010.

Je remercie Dario Fo, Franca Rame et Jacopo Fo qui ont généreusement autorisé la reproduction des documents en leur possession et/ou visibles sur le site www.archivio.francarame.it/ (photographies ou photogrammes à partir des captations). Les auteurs des photographies n'étant pas identifiés, je regrette de n'avoir pu les solliciter.
} 
parole proférée avec le geste par lequel l'acteur construit son rapport kinésique avec les objets, objets qu'il fait exister dans la perception du spectateur en les manipulant ou en mimant leur utilisation. Dans le Manuale minimo dell'attore (Manuel minimum de l'acteur $^{8}$ ), qui rassemble le texte des conférences-spectacles données dès 1987 par Dario Fo à l'intention d'apprentis comédiens, et qui doit sans nul doute être considéré comme le recueil le plus complet de ses déclarations sur sa conception du texte, du jeu et de la mise en scène, aucune entrée dans le « Glossaire des termes usités et inusités au théâtre », aucun titre de paragraphe rythmant les « Journées » ne synthétise les diverses modalités du rapport à l'objet.

Mais la leçon consacrée au monologue en grammelot sur La fame dello Zanni (La Faim du Zanni), ce paysan bergamasque ruiné par la chute des prix alimentaires et devenu presque esclave dans la Venise du $\mathrm{XV}^{\mathrm{e}}$ siècle, constitue une poétique en acte: Dario Fo choisit de décrire et d'effectuer le geste par lequel un objet matériellement absent sur la scène est rendu présent dans le regard du spectateur au moment précis où ce geste s'allie à une parole faiblement dénotative. Rappelons que le grammelot - auquel Fo, selon le traitement habituel qu'il affecte à ses sources, résolument non dignes de foi mais toujours fascinantes comme il l'indique lui-même, se plaît à attribuer une origine moliéresque -, est une palabre onomatopéique, parsemée de quelques mots attestés et destinée à fonder, par la combinaison de phonèmes récupérés, imités ou inventés à partir d'une aire linguistique variable, l'image sonore et la cadence d'une langue étrangère ou d'un dialecte exotique. Le signifié étant insuffisamment porté par le verbe, c'est à l'expressivité du corps, parfois même dans le jeu avec masque, qu'il appartient de construire mentalement la chose non dénommée : l'objet et son usage dans une situation donnée. L' « art » du mime - non pas en tant qu'esthétique mais en tant que métier - est ainsi défini en termes de technique physique, de rythme et de vocalité. Car il importe ici de préciser que, proche sur ce point de son ami Jacques Lecoq ${ }^{9}$, Dario Fo donne du mime une définition extensive, qui est celle originelle : « l'acteur total, qui sait se servir de la voix, du geste, du chant et de l'acrobatie ${ }^{10}{ }$. La pratique langagière et gestuelle de Fo serait en ce sens une confirmation, au théâtre, des théories de la linguistique cognitive selon laquelle le langage est embodied (incarné) et contextualisé dans un espace mental global et cohérent. Un dessin dans le Manuel représente d'ailleurs le spectateur, assis dans un fauteuil ${ }^{11}$, les bras croisés, le crâne

\footnotetext{
8 Une traduction de ce texte a été faite sous un titre qui en modifie quelque peu la portée puisqu'il l'inscrit sous le signe du gai saber des troubadours, plutôt que sous celui de la tradition contestataire du giullare tel que, à partir d'une interprétation très personnelle des travaux de certains médiévistes (Chiara Frugoni, notamment), le définit Fo. Voir Dario, Fo, Le Gai Savoir de l'acteur, traduction française de Valeria Tasca, Paris, L'Arche, 1990.

Jacques Lecoq (1921-1999), initié à l'art du mime par Étienne Decroux, élève de Jean Dasté qui lui fait découvrir le travail du masque, a participé en Italie à partir de 1948 aux recherches d'Amleto Sartori sur «le masque neutre », à la création de l'École du Piccolo Teatro de Milan puis, aux côtés de Franco Parenti et Dario Fo, à l'élaboration d'un «théâtre du geste » qui se souvient de la Commedia dell'Arte. En 1956, il rentre à Paris et fonde une école internationale destinée à former tous les artistes du théâtre et notamment des comédiens aptes à développer, sur la base de l'improvisation, toutes les ressources corporelles d'une dynamique de jeu.

10 Dario, Fo, Manuale minimo dell'attore, Turin, Einaudi, 2001 ( $1^{\text {ère }}$ éd. 1987), p. 341. Je traduis à partir de l'édition italienne.
}

11 Ibid., p. 65. 
formé d'une caméra : ce qui constitue la portée de son regard (c'est-à-dire de son attention) en «cadrage ». L'acteur, par la mobilité de son corps, par une gestualité qui crée des objets - lesquels la contraignent en retour -, oriente la concentration du spectateur sur les divers éléments du récit, du «plan moyen large» au «plan rapproché », du «premier plan » au «très gros plan ». La narrativité, dans La Faim $d u$ Zanni, est assez faible, tout comme dans nombre de pièces qui sont un agencement de sketches plutôt qu'une fable fortement développée - de Gli arcangeli non giocano a flipper (Les Archanges ne jouent pas au flipper) en 1959, concaténation de tromperies et farces à l'encontre d'un naïf, jusqu'à Claxon trombette e pernacchi (Klaxon, trompettes et pétarades) en 1981, où se succèdent les quiproquos -. Le discours contextualise et sollicite l'imaginaire du spectateur bien plus qu'il ne dénote puisqu'il est proféré en grammelot. Mais la brutale concrétude des gestes et des objets, nommés et mimés, que mange le Zanni dans la "réalité" ou dans son rêve scéniques - son œil devenu œuf, ses tripes-saucisses, la polenta mise à cuire dans la poêle, le morceau de bœuf, son doigt-os de poulet, le bâton, et la mouche qui achève ce «gueuleton »- mêle au comique de la méprise la valeur tragique de l'effet de réalisme : l'objet a permis de fonder un langage en lequel le peuple, interdit de parole par la culture dominante, retrouve sa force d'expression. Le spectateur perçoit la dénonciation inscrite dans la déconstruction de l'illusion théâtrale - les objets manquants sur la scène sont ainsi les marques de la faim réelle, l'expression de l'entité "Faim"12 - et il peut alors, lors de son retour effectif au réel du monde, dans sa réflexion personnelle, transférer sur les problèmes de la Cité la force d'indignation qui sous-tend le spectacle.

Ainsi convient-il, dans une perspective résolument dynamique et fonctionnelle, d'échapper à la fascination actorielle pour percevoir, lors de la contemplation spectatorielle autant que dans l'analyse critique, l'articulation des signes et des systèmes sur la scène, en une totalité qui est complexe et stratifiée, même si la progressivité de sa constitution est, comme toujours au théâtre, masquée par la simultanéité de tous les éléments lors de la représentation ${ }^{13}$. C'est donc au

12 Il faut noter que, dans la description que fait Dario Fo de la progressivité de l'attention du spectateur, sans sautes et par concentration, l'achèvement du rôle de l'objet sur le très gros plan de la mouche constitue un paroxysme d'intensité mais surtout de qualité. L'adoption par Dario Fo de la terminologie filmique, rendue légitime mais surtout nécessaire par le cerveau-caméra du spectateur, permet de considérer comme pertinente ici la remarque de Gilles Deleuze sur le gros plan au cinéma : « le gros plan n'arrache nullement un objet à son ensemble dont il ferait partie, dont il serait une partie, mais, ce qui est tout à fait différent, il l'abstrait de toutes coordonnées spatio-temporelles, c'est-à-dire il l'élève à l'état d'Entité ». Gilles, Deleuze, L'Image-mouvement, Paris, Les Éditions de Minuit, 1983, p. 136.

13 La consultation du site $\underline{w w w . a r c h i v i o . f r a n c a r a m e . i t ~ p e r m e t ~ d e ~ r e n d r e ~ h o m m a g e ~ a ̀ ~ l ' e ́ c l a i r a g e ~}$ génétique que permet le considérable travail d'archivage réalisé par Franca Rame. Le processus de création, d'une durée très variable, y est documenté par les notes manuscrites (idées de scénario, canevas, conception d'un personnage ou d'un argument en réaction à des faits politiques ou sociétaux, notes de lectures, notations gestuelles...), les tapuscrits (états textuels différents), une abondante iconographie d'images fixes (dessins d'un costume ou d'un visage qui définissent un rôle, plan d'implantation ou croquis de blocking apte à condenser la signification d'une scène, peinture d'un fond de scène... ), des captations permettant d'apprécier les variations de la mise en scène et du jeu... 
sein de cet ensemble que l'objet théâtral doit être défini dans sa nature, son statut et surtout dans la fonction qui détermine sa portée sémantique.

\section{Le refus de l'imitation au théâtre}

L'examen de la leçon consacrée à La Faim du Zanni, la lecture du texte et la vision de l'enregistrement vidéo confirment l'importance du rapport objet/espace dans la constante stimulation de l'attention du spectateur. Car le projet fondateur dans l'écriture et la pratique scénique de Dario Fo et Franca Rame est, comme ils le répètent souvent sous des formes légèrement différentes mais en conservant toujours la même image, d' « ouvrir tout grand le cerveau du spectateur » («spalancare il cervello ») en lui parlant des problèmes vrais et actuels de sa vie et de son temps. C'est par la représentation, lorsque se noue in praesentia la communauté théâtrale, que le travail de Fo et Rame atteint son efficacité militante en provoquant l'ébranlement qui vise à faire de chaque spectateur un agent de sa propre vie et non un acteur du spectacle ${ }^{14}$. Il faut rappeler ici, pour éviter toute équivoque vu que Dario Fo insiste sur la nécessaire «implication physique du spectateur» ( «coinvolgimento anche fisico ${ }^{15} »$ ), qu'il n'y a, dans la dramaturgie de Fo, aucune intervention de type happening mais tout au plus la gestion, avec le maintien du spectateur en position de récepteur ne conditionnant la scène que par son système d'attentes, d'éventuels incidents préparés ou non et survenant pendant la performance; et c'est seulement dans les débats qui, éventuellement, suivent la représentation que le spectateur trouve son lieu d'expression effective. De cette vocation éducative du théâtre de Fo découle néanmoins la constante nécessité d'un espace de déplacement, de mouvement physique et mental, où puisse s'opérer l'arrachement à la fabula et au plat mimétisme naturaliste des objets scéniques.

Certes, l'iconicité de l'objet doit provoquer son identification par le spectateur, mais seulement afin que les codes d'usage de cet objet dans le réel soient mobilisés pour être aussitôt démontés et contredits par la pratique scénique : le quatrième mur de scène est abattu mais la conscience de la différence entre le vrai et ce qui "semble vrai" demeure forte. «Le théâtre est fiction de la réalité et non imitation $^{16} »$, affirme Dario Fo. La scénographie laisse donc peu de place à la vocation secondaire de l'accessoire, qui ne saurait demeurer confiné dans une fonction référentielle ou théâtrale traditionnelle. Si, lorsqu'en 1957-1958 au début de sa carrière, dans la farce I cadaveri si spediscono e le donne si spogliano (Expédition des cadavres et déshabillage des femmes), pièce qui se passe dans un atelier de costumes de théâtre où le paravent servant à se dévêtir dénote avec évidence son utilité, Dario Fo reprend cet expédient dramatique fréquent dans le

14 Dario Fo intègre la leçon brechtienne sur la forme épique du thêâtre en la réinterprétant. Toute identification au personnage doit être impossible: ce à quoi concourent d'ailleurs l'interprétation par un même acteur de plusieurs personnages, le travestissement à vue ou visible, l'adresse au spectateur...

15 Dario, Fo, Manuale..., op. cit., p. 101.

16 Ibid., p. 235 : «Il teatro è finzione della realtà, non imitazione. » 
vaudeville $^{17}$, il en amplifie aussitôt l'usage de sorte que le procédé devienne manifeste et acquière une signification symbolique :

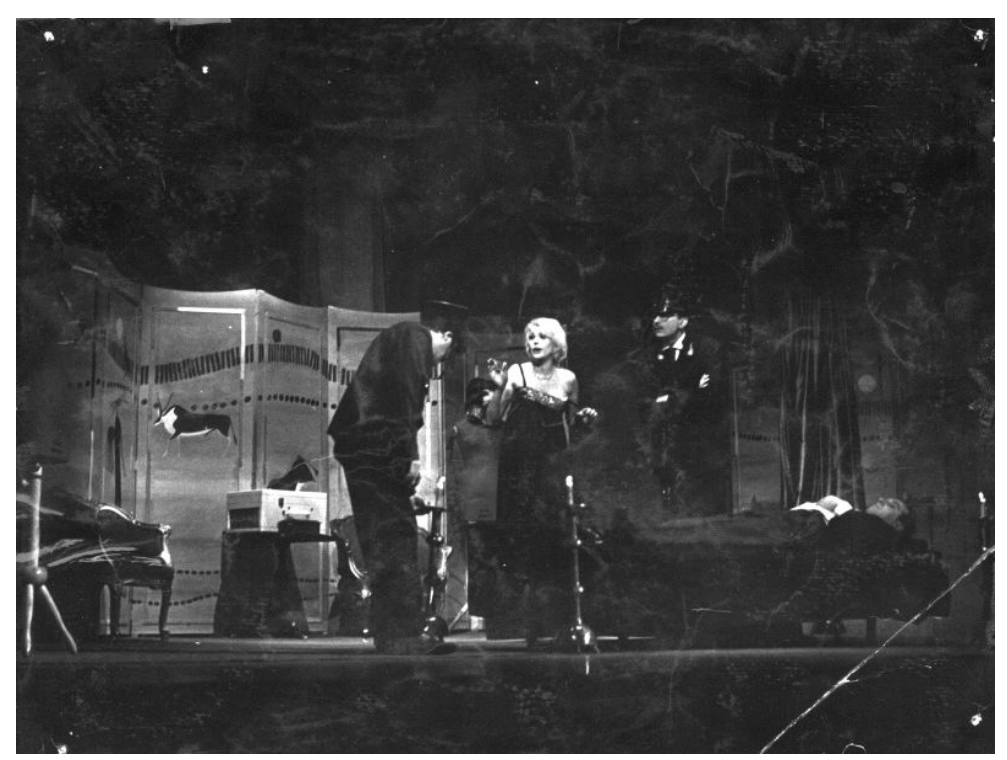

\section{I cadaveri si spediscono e le donne si spogliano, in Ladri, manichini e donne nude, (C) /archivioforame/LMDN/1958/FOTO/001380/001380-010m}

L'espace scénique s'en trouve certes ouvert au comique de situation (quiproquos, équivoques, apparitions et disparitions, travestissements) mais quatre paravents, et non plus le paravent, superposent les niveaux du jeu sur l'identité sexuelle, les rapports homme/femme, la multiplication des cadavres. De sorte qu'au rire spontané que déclenche la farce, contaminée en outre par le néo-policier et le macabre, se mêle l'inquiétude liée à la corrosion des relations humaines.

On comprend que le détournement de l'objet de son usage ordinaire ou conventionnel est bien un élément de la théâtralité globale construite par Fo, et non point seulement une caractéristique de son jeu personnel en tant qu'acteur, lorsque l'on considère la mise en scène du Médecin volant de Molière ${ }^{18}$ réalisée pour la troupe du Français. À la scène XV, le balai qui supporte la perruque et le manteau de Sganarelle (incarné par Christian Blanc) dépasse sa qualité quotidienne d'objet rappelant la situation sociale du serviteur déguisé en médecin, pour fonder l'action sur la scène : il exalte la légèreté aérienne du personnage volant de porte en fenêtre

\footnotetext{
17 On rappellera qu'en cette même année 1957 Franca Rame joue, à Rome au Teatro Arlecchino, Mais n'te promène donc pas toute nue! de Feydeau et que l'usage du paravent dans ce vaudeville, comme auparavant chez Labiche, n'a pu manquer de rappeler à Dario Fo la force de cet accessoire pour créer un comique de situation, en permettant que deux personnages soient simultanément sur la scène, chacun ignorant la présence de l'autre. La pièce a d'ailleurs été jouée en même temps que trois farces de Dario Fo dans le spectacle intitulé Ladri, manichini e donne nude (Voleurs, mannequins et femmes nues).

18 Molière, Le Médecin volant et Le Médecin malgré lui, mise en scène de Dario Fo, Paris, Comédie-Française (salle Richelieu), création le 9 juin 1990.
} 
et jouant du travestissement pour tenter de dissimuler son mensonge (la soi-disant existence de son frère jumeau). Puis une corde, qui permet à Sganarelle de sortir de la maison dans laquelle l'a enfermé Gorgibus, confirme l'agilité mentale par laquelle se manifeste l'aptitude du valet à tromper ce bourgeois lourdaud; mais, lorsque le stratagème est découvert, le mouvement se fige et la fonction symbolique de l'objet, énoncée et montrée sur la scène, se transforme : la corde du cerf-volant devient celle du pendu, châtié à mort. Un autre exemple, dans cette même mise en scène, confirme l'élargissement de la portée sémantique des objets. À la scène IV, l'adjonction par Dario Fo, au regard de ce qu'indique le texte canonique de Molière (celui édité dans la Pléiade, par exemple), de la longue verbalisation et manipulation des objets médicaux par Sganarelle (la bougie, la cuiller, la loupe, le clystère, le pot de chambre) amplifie la crédulité de Gorgibus et aiguise la satire contre l'autorité des médecins en reprenant notamment le motif, traditionnel dans la farce, de l'urine goûtée, bue, et crachée ou avalée. Néanmoins, dessinés par le metteur en scène sur les deux gros carnets avec lesquels il arriva aux répétitions, ces objets n'épuisent nullement leur signification dans le rire immédiat du spectateur. Si leur manipulation semble d'abord évoquer l'habileté d'un prestidigitateur et rappeler par là que le théâtre tel que le conçoit Dario Fo n'installe aucune hiérarchie entre toutes les formes du spectacle, qu'elles soient dites nobles ou populaires, elle renvoie aussi à la façon dont, au XVII ${ }^{\mathrm{e}}$ siècle, le «théâtre des opérateurs » aidait les bateleurs à vanter les vertus des produits et objets médicaux. Car on sait que ces vendeurs, installant leurs tréteaux dans les endroits les plus passants des villes ou dans les foires, faisaient parfois soutenir leur boniment par des comédiens débutants, jouant des parades et des farces; et l'on a même retenu des joutes littéraires de l'époque que Molière, peut-être, aurait commencé ainsi sa carrière d'acteur ${ }^{19}$. L'insistance de Dario Fo sur ces objets médicaux prétendument miraculeux serait alors à la fois un discours sur l'unicité complexe et diverse du spectacle scénique et un hommage à son maître Molière.

Toutefois, l'emploi de l'objet comme fondement-certes formellement modulable - d'une dramaturgie italienne, revisitant la tradition et innovant tout à la fois, caractérise également le travail de Dario Fo metteur en scène, costumier et décorateur pour le théâtre lyrique, soit dans ses mises en scène d'opéra ou opéra bouffe de Rossini. On évoquera, dans Il barbiere di Siviglia ${ }^{20}$ (Le Barbier de Séville), le groupe de mimes portant des costumes et des masques de la Commedia dell'Arte qui jonglent, exécutent des acrobaties ou dansent pendant l'air de Figaro «mon Figaro est frère d'Arlequin », a affirmé Dario Fo lors d'une interview au Corriere della sera -. Ou encore Rosina qui, proclamant sa désobéissance dans la cavatine de l'acte I, joue de son tambour à broder et de sa pelote de fil comme avec la raquette et la balle d'une sorte de jokari, affichant par ce détournement de l'objet (de l'intimité du foyer à l'espace extérieur, du travail au jeu, de l'immobilité au mouvement, du XVIII ${ }^{\mathrm{e}}$ siècle au XX $\mathrm{XX}^{\mathrm{e}}$ ) la scandaleuse inadéquation entre sa jeunesse

19 C'est du moins ce que laisse supposer le Boulanger de Chalussay dans l'Élomire hypocondre (acte I, scène 1), le pamphlet hostile à Molière qu'il publia en 1670, après l'affaire du Tartuffe.

Spectacle créé en février 1987, De Nederlandse Opera d'Amsterdam ; repris à l'Opéra de Paris pour douze représentations, du 13 juin au 7 juillet 1992. 
et les prétentions amoureuses de son vieux tuteur. Enfin, pendant l'air de la Calomnie (I. 8), ce sont la dimension et la multiplication des objets, réunis dans l'espace en une même totalité visuelle de forme et de mouvement (une rotondité tournoyante qui se plie et se déplie) et aussi de couleur (la menaçante luminosité d'une étoffe grège), qui matérialisent la propagation du «vent léger de la calomnie »: la scène est parcourue de plus en plus rapidement par le déplacement d'un immense parasol derrière lequel se cache d'abord Don Basilio puis, en un processus de réduction et de multiplication, par le glissement d'hommes en redingote noire qui sautent en tumulte, la cervelle étourdie, jusqu'à l'acmé visuel et sonore de l'expansion («Et elle [la calomnie] produit une explosion / Comme un coup de canon / Comme un coup de canon »), au terme de laquelle les pauvres calomniés rampent à genoux et « par grand malheur s'en [vont] crever $»^{21} \ldots$

Mais pour en revenir au théâtre de Dario Fo, par et avec Dario Fo, dans Les Archanges ne jouent pas au flipper par exemple, c'est, au sein d'une pièce structurée de façon apparemment traditionnelle en trois actes et huit tableaux, un élément du décor qui est détourné de sa fonction illustrative pour fonder la satire. Reprenant le motif, classique depuis l'Amphitryon de Plaute, du double, du déguisement et du quiproquo, Dario Fo développe l'histoire des mésaventures d'un "naïf" soumis à toutes sortes de tromperies de la part de ses camarades (un faux mariage, notamment) et victime de la société. Inscrit par erreur à l'état-civil comme «chien braque », ce personnage - incarné par Dario Fo lui-même comme tous les personnages de son théâtre décalés par rapport à la "normalité" : le naïf, le fou, le marginal... - tente de récupérer son identité humaine perdue. Dans une scène au rythme très rapide, les cinq employés du ministère romain concerné, coiffés d'une espèce de calotte de clown chauve posée sur une chevelure touffue, ne cessent d'ouvrir et de fermer leurs guichets pour en interdire l'accès au demandeur. Par l'accélération du mouvement d'ouverture et de fermeture, par la répétition de la chute verticale du rideau qui coulisse brusquement comme une guillotine au moment où les employés doivent apposer le timbre du service sur des documents, l'objet développe une vitalité improductive, destructrice et finalement mortifère.

21 Une analyse kinésique très précise montrerait l'exacte correspondance entre les paroles du livret de Cesare Sterbini et la gestuelle des acteurs, qui les explicite et les ponctue en chaque phase de la progression. 


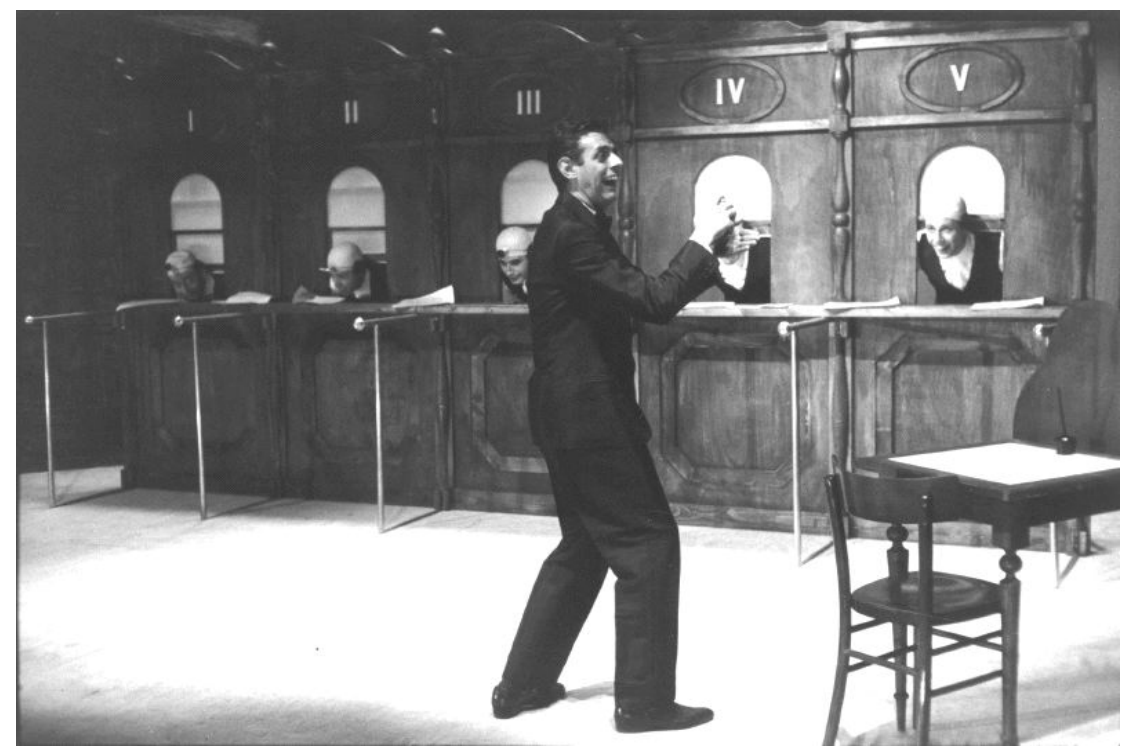

\section{Gli arcangeli non giocano a flipper, (C)} larchivioforame/ARCA/1959/FOTO/001388/001388-008m

Le gag, directement issu du burlesque des origines-les transformations de Fregoli dans les attractions qui ouvraient les spectacles de variétés ou les numéros comiques des premiers films muets (le slapstick et le running gag)-confère à l'objet un excès d'énergie, comme lorsque Buster Keaton, dans Moonshine ${ }^{22}$, fait sortir d'une seule et même voiture des dizaines d'officiers. Le comique, fondé sur la dissonance du rapport liant l'homme à l'objet, se fait dénonciateur: le tragique affleure, celui d'une humanité broyée par la civilisation bureaucratique. Mais il importe de signaler que, si la parodie visuelle et la surcharge compulsive convoquent en le spectateur le souvenir d'un certain langage gestuel propre au clown et fréquemment mis en jeu dans la dramaturgie de l'objet au $\mathrm{XX}^{\mathrm{e}}$ siècle qu'il s'agisse du chapeau de Vladimir ou des chaussures d'Estragon dans En attendant Godot ou même, par glissement de la notion de réitération, de la prolifération des chaises chez Ionesco -, l'application de ce jeu par Dario Fo aux seuls aspects de la vie des hommes qu'il conteste sociologiquement et politiquement interdit d'y lire toute insignifiance radicale du réel ou absurdité inhérente à la condition humaine. Et surtout, si le délire gestuel engendré par l'objet, arraché à tout mimétisme et à toute logique référentielle, provoque comme un inquiétant élargissement des frontières connues et admises du réel, l'appel à un ancrage dans ce réel que Dario Fo lance au spectateur, citoyen hors du théâtre, pour une lutte volontaire et concrète n'en est que plus fort.

22 Moonshine, film de et avec Roscoe 'Fatty' Arbuckle (1918) : seuls quelques extraits ont été conservés à la Cinémathèque de Rome, le film complet ayant disparu ; certains sont néanmoins consultables sur le web. 


\section{La scène et l'accès à un "surréel" fictif}

Dans la première période du théâtre de Dario Fo, lorsque le lien au théâtre de variétés est encore très proche, la déroutante étrangeté de certains objets scéniques demeure contenue dans le cadre de l'effet comique : qu'il suffise d'évoquer les dragées qu'ingère un personnage et qui font aussitôt entendre le bruit d'une explosion dans son estomac (Expédition des cadavres...). Précisons qu'il ne s'agit nullement de l'unheimlich décrit par Freud, ni même de la surprise éprouvée devant les tableaux de De Chirico, ce peintre que Dario Fo admire tant, quand la familiarité du visible se casse pour laisser affleurer la réalité psychique des objets. Ce qui est construit par Dario Fo, auteur-acteur et metteur en scène en ce cas, est un retrait par rapport à l'action afin que soit constitué un espace de perception inédit pour le spectateur, où l'effet de reconnaissance est effacé et modifié aussitôt que produit: là, s'inscrit l'épisation ${ }^{23}$ du théâtre qui permet l'exercice critique. La "distanciation" est assurée par l'affichage d'un point de vue insolite sur ce qui est montré sur scène ainsi que par la variabilité de ce point de vue.

Dans Settimo, ruba un po' meno (Septième commandement: Tu voleras un peu moins), en 1964, la discordance initiale - un personnage, joué par Franca Rame, de croque-mort femme nommé(e) Enea (la terminaison en -a est sentie comme un féminin !) - ouvre à la perturbation généralisée des paradigmes d'appréhension des "effets (scéniques) de réel": le spectateur, dérouté, incapable de transférer vers la scène ses codes usuels de déchiffrement du réel quotidien, devient réceptif à un regard autre. L'objet, hybridisé, arraché à sa fonction et parfois même à son essence (l'inanimé), recèle alors des fonctions pragmatiques inédites. Outre son utilisation dans le gag banal (Énée arrose par distraction les pieds d'un de ses compagnons), l'arrosoir sert à donner de l'eau à des fleurs faites de fer. Le geste de l'acteur détournant l'objet de son usage obligé, installe ainsi une confusion des règnes, le végétal et le minéral : et c'est, subrepticement, l'ordre même de la nature qui se trouve mis en cause. Puis, au-delà du comique de situation le plus convenu (l'ensevelisseur enseveli), le cercueil, où se joue d'abord une parodie de Résurrection, devient ensuite le lieu curateur où, soi-disant sur prescription de son médecin, le conseiller commercial «catafalqu(o)phobe » (feretrofobo) doit s'étendre pour guérir de son obsession tout inédite, qui apparaît comme une sorte de variante composite de nécrophobie, nyctophobie et thanatophobie: par la gestuelle de l'acteur, le cercueil se mue alors en une baignoire, où il faut entrer précautionneusement pour supporter le contact froid, puis en une barque que deux cierges pris sur le corbillard et servant de rames font reculer sur l'eau.

23 Voir Patrice, Pavis, Dictionnaire du théâtre, Paris, Éditions sociales, 1980, p. 150. 


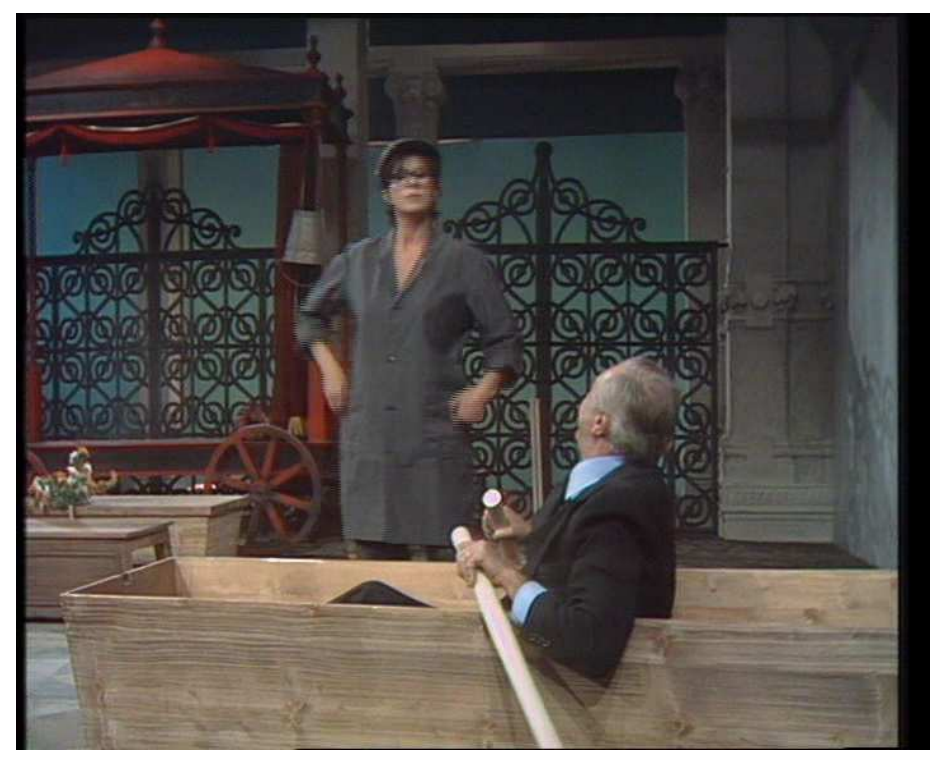

Settimo, ruba un po' meno, () Dario Fo, Franca Rame et Jacopo Fo, captation à partir de l'enregistrement RAI, Milan, Palazzina Liberty, 1977

Le cercueil redevient ensuite une bière ordinaire où le mari s'étend et feint la mort pour provoquer une crise cardiaque fatale en son épouse et s'en voir libéré. La gestion scénique du couple et du triangle caractéristiques du vaudeville se double ainsi d'une critique de la psychiatrie, de la religion, de la civilisation des loisirs, du mariage enfin, c'est-à-dire de certaines constructions psychiques grâce auxquelles se meublent les vies. Et l'on notera, dans cette scène, l'indissoluble union, dans l'action scénique, du geste, de l'objet et de l'invention langagière (les néologismes). À un autre moment, montant sur le corbillard présent en scène, les quatre croque-morts hommes se parent d'un masque de carnaval (un blanc visage de vieille poupée) et chantent l'hymne sacré à la gloire des prostituées, phares de la civilisation, véritables Croisé(e)s d'amour et de charité. S'alignant face au public, montrant la discordance entre leur visage masculin et leur masque féminin, ils lancent les jambes comme les danseuses canailles d'un provocant cancan: le corbillard est ainsi transformé en planches de Caf' Conc'. 


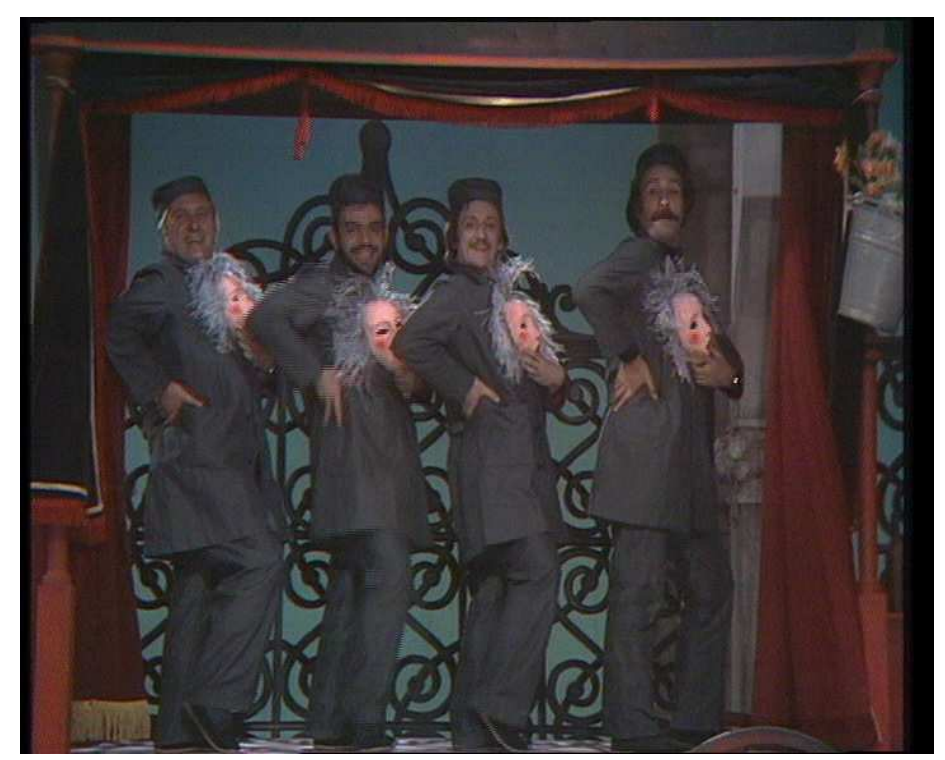

Settimo, ruba un po' meno, () Dario Fo, Franca Rame et Jacopo Fo. Captation à partir de l'enregistrement RAI, Milan, Palazzina Liberty, 1977

Or cette métamorphose affectant cet objet là, le corbillard, alors même qu'elle rappelle les origines du théâtre de pur divertissement, est aussi la relecture visuelle et inversée de l'incident de tournage - l'omnibus changé en corbillard - qui fut, si l'on en croit l'histoire ou la légende, fondateur du trucage au cinéma «dans sa voie théâtrale spectaculaire », selon la formule qu'adopta Méliès, alors prestidigitateur et propriétaire du Théâtre Robert-Houdin à Paris, pour définir au printemps 1898 sa conception de cet art naissant ${ }^{24}$. La mise en abyme d'un art dans l'autre et la contamination des formes diverses du spectacle - prestidigitation, théâtre, variétés, film - affirment ainsi le refus en Dario Fo de la catégorisation en genres et du rituel spectatoriel figé du théâtre bourgeois, pour promouvoir la vocation globale d'un théâtre qui divertit et éduque tout à la fois.

C'est à partir de cette densité sémantique que l'objet scénique, fût-ce dans son existence théâtrale première de costume ou d'accessoire du décor, peut fonder une féroce dénonciation sociétale (le vol politique, la spéculation immobilière, la confiscation anormale des pouvoirs), la satire des vices moraux (l'hypocrisie et la compromission), les choix éthiques. Aussi faut-il considérer, par exemple, que c'est la variation du costume d'Enea qui constitue, dans Septième commandement : Tu

24 Georges, Méliès, «Les vues cinématographiques », in Annuaire général et international de la Photographie, Paris, Plon, 1907 ; à présent in Georges, Sadoul, Georges Méliès, Paris, Seghers (Cinéma d'aujourd'hui), p. 106-107: «Un blocage de l'appareil dont je me servais au début (appareil rudimentaire, dans lequel la pellicule se déchirait ou s'accrochait souvent et refusait d'avancer) produisit un effet inattendu, un jour que je photographiais prosaïquement la place de l'Opéra : une minute fut nécessaire pour débloquer la pellicule et remettre l'appareil en marche. Pendant cette minute, les passants, omnibus, voitures avaient changé de place, bien entendu. En projetant la bande, ressoudée au point où s'était produite la rupture, je vis subitement un omnibus Madeleine-Bastille changé en corbillard et des hommes changés en femmes. Le truc par substitution, dit "truc à arrêt" était trouvé $[\ldots]$ » 
voleras un peu moins, non pas la structure porteuse d'une action nullement linéaire ou progressive mais plutôt le fil conducteur du sens. La croque-mort naïve qui, initialement bernée par la fable des prostituées rédemptrices de l'humanité, s'était habillée en tapineuse (une robe aguichante, un unique bas de soie qu'elle avait enfilé sur son bras, des talons aiguille avec lesquels elle se tordait les pieds, une perruque rousse et un parfum qui attirait même les chats) résume le sens final de la pièce en se dépouillant de son "costume", marquant par là son refus des compromissions et de la corruption dans une société de l'apparence et de l'argent que son nouvel habit lui avait permis de pénétrer. Tandis que, coiffés d'une calotte surmontée d'une hélice qui les fait «toujours aller dans le sens du vent», les représentants de tous les pouvoirs institués (le Directeur, le Commissaire, le Juge, l'Excellence, le Professeur) et deux Fous chantent et dansent les couplets ${ }^{25}$ de l'italiote, soit l'Italien moyen qui a béatement subi un lavage de cerveau politique ${ }^{26}$, Enea affirme sa liberté et la conscience acquise :

Enea (amère) Oui...oui... Moi, vraiment, je suis très heureuse. (Elle enfile la vareuse sur sa robe de prostituée) Et même... je dois vous remercier parce que, sans vous en apercevoir, moi aussi vous m'avez trépanée, mais dans le bon sens... D'un seul coup, vous m'avez ouvert le cerveau tout grand! (Elle enlève sa perruque et la lance à un des Fous). Merci, Excellence. (Elle enlève ses sandales et enfile ses bottes) [...] Je repars là d'où je viens. Moi, vous ne me ferez pas le coup de me mettre une hélice sur la tête, ni des lunettes vertes pour me faire manger de la paille en me faisant croire que c'est de l'herbe. ${ }^{27}$

Ici, il ne s'agit pas seulement du changement de costume à vue qui, comme le changement de décor à vue dans le théâtre de Dario Fo, abat le quatrième mur de scène et détruit la séparation scène/coulisses pour livrer au spectateur les règles de fabrication du spectacle. Il n'importe pas davantage, par une exhibition du travail théâtral, de marquer la différence de statut entre l'acteur et le(s) personnage(s) interprété(s). De ces données fondatrices de la distanciation critique, le spectateur a déjà la pleine compréhension. Fo vise plutôt ici une sorte de complétude sémantique

25 On notera que l'utilisation du song (au sens brechtien du terme) comme moyen de distanciation s'accompagne toujours chez Fo d'une manipulation d'objets qui dénonce la prétendue unanimité du groupe et qui, théâtralement, articule l'action politique et les aspects divertissants du théâtre de variétés.

26 Le référent appelé par la «trépanation» mise en scène varie selon les dates de représentation et d'édition de la pièce : de la chape de plomb morale et politique installée par la Démocratie Chrétienne (dans Septième commandement I, en 1964) à la corruption du politique par le pouvoir économico-médiatique sous Berlusconi (dans Septième commandement I, en 2006). Le processus et les mécanismes de corruption demeurant inchangés, ils peuvent être presque identiquement mis en gestes et en images sur la scène.

Dario, Fo, Settimo : ruba un po’ meno, acte II, in Teatro, p. 209 : « ENEA (amara) Sí.. Sí... Io, poi, sono felicissima. (S'infila la palandrana sopra l'abito da prostituta) Anzi... Vi devo ringraziare perché, senza accorgervene, avete trapanato anche me, ma nel senso giusto... In un colpo solo, mi avete spalancato il cervello! (Si toglie la parrucca e la getta a uno dei Pazzi) Grazie, eccellenza. (Si leva i sandali e si infila gli stivali). [...] Torno da dove sono venuta. A me non ce la farete a mettermi l'elica in testa, né gli occhiali verdi per farmi mangiare la paglia e farmi credere che sia erba !» 
de l'acte scénique, où l'alliance entre la gestuelle de l'acteur et la verbalisation du geste par lequel celui-ci manipule l'objet qui recouvre son corps accède à la fonction de conclusion de l'action dans la pièce : la fabula a diverti, mais c'est par le rapport que l'acteur établit entre son corps et l'objet, dénonçant ainsi l'état du monde, que le spectateur est «éduqué ».

\section{L'invention des objets scéniques}

La densification sémantique des objets et le systématique effet d' "étrangification" dont ils sont affectés dans la dramaturgie de Dario Fo (pour reprendre le néologisme proposé par Anne Ubersfeld en guise de traduction du Verfremdungseffekt brechtien) s'étendent très nécessairement à l'ensemble du dispositif scénique. Ainsi le décor participe-t-il littéralement à l'action, par exemple au second acte de Klaxon, trompettes et pétarades, lorsque les meubles se déplacent et se groupent pour lire de plus près la publication, sur un journal, d'une lettre que Gianni Agnelli, enlevé par des terroristes selon l'intrigue de la pièce, aurait écrite à l'intention de Spadolini. La stupeur des objets scéniques, arrachés à leur immobilité naturelle par «l'invraisemblable », souligne évidemment, par contraste et transfert, la réalité de l'Histoire : la passivité du gouvernement démocrate chrétien lorsque le chef du parti, Aldo Moro, fut en mars 1978 enlevé et séquestré par les Brigades Rouges et, par de nombreuses lettres, implora en vain les représentants de l'État de négocier avec ses geôliers avant d'être assassiné.

L'exaspération de la fiction scénique par un auteur qui ne cesse de répéter, notamment dans des prologues où il s'adresse directement au public, que « le faux, au théâtre, est plus digne de foi » que la communication directe de la réalité désormais organisée, selon la stratégie médiatique du pouvoir, pour produire dans le peuple « un encéphalogramme plat ${ }^{28}$ »-, qui affirme constamment que les hommes politiques gouvernant l'Italie lui "volent" les idées les plus invraisemblables de ses pièces sans pour autant lui payer des droits d'auteur, stimule, de fait, la participation critique du spectateur. Des objets scéniques inédits et totalement improbables, fruits de l'imagination et de l'ironie, activent ainsi, par renversement des schémas et images standard, la réflexion sur la réalité qu'ils visent à signifier. On ne saurait bien sûr les citer tous ni même évoquer les modalités diverses selon lesquelles ils sont, selon les cas, verbalisés et/ou montrés. Dans Klaxon, trompettes et pétarades, lorsqu'un quiproquo a amené des chirurgiens à reconstituer sans le savoir sur le corps blessé de l'Avvocato Agnelli, le patron de Fiat, le visage d'un de ses ouvriers, ce sont divers objets qui manifestent visuellement cette conjonction impensable de l'exploiteur et de l'exploité : une toile d'araignée de bandages mus par des fils laisse apparaître le visage de la créature dénommée Sosie tandis que le dialogue évoque les films d'horreur des années trente (Boris Karloff dans Frankenstein ou La Momie); un masque permet à ce Sosie recousu de partout d'ingérer un bouillon grâce à tout un système de tuyaux, puis un pot-au-feu par le moyen d'un hachoir à viande installé sur son crâne : les bruits de manducation et de déglutition sont une sirène de navire, un blues puis un rock joués par une clarinette... 


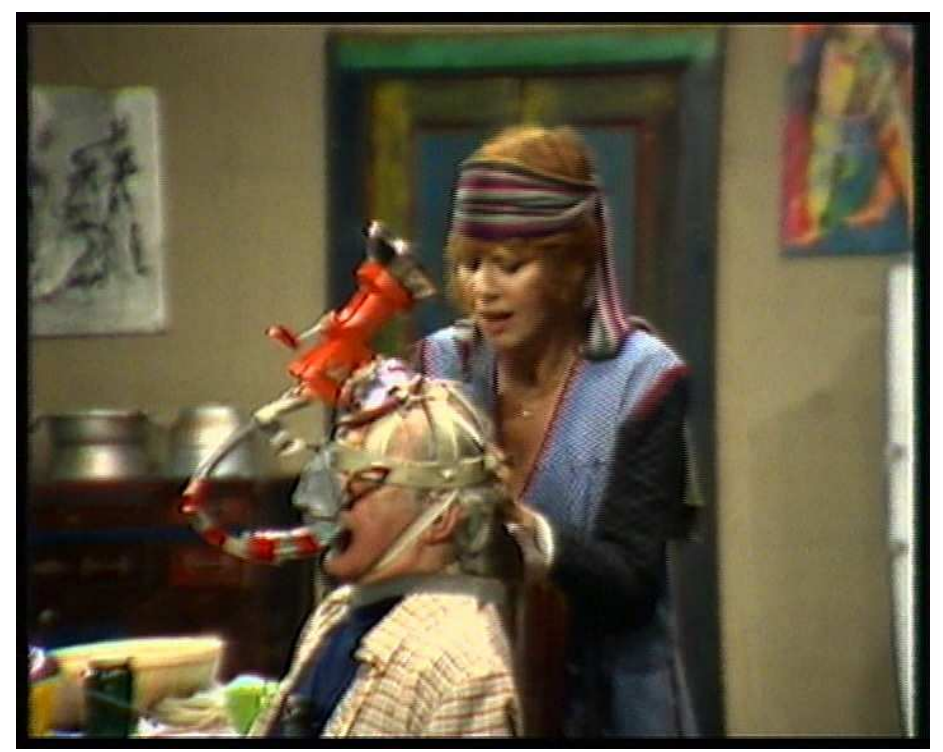

Claxon, trombette e pernacchi, , (C) Dario Fo, Franca Rame et Jacopo Fo, Captation à partir de l'enregistrement au Teatro Tenda de Rome et au Teatro Politeama de Naples

Le grotesque et l'hybridation qui ont porté à l'invention de ces objets affichent la monstruosité du réel. Il est donc logique que, dans l'imaginaire et la volonté militante de Dario Fo, l'anomalie ${ }^{29}$ de la vie politique italienne - le continuel enrichissement illégal de Berlusconi qui assure son impunité en faisant voter par ses avocats, députés élus de son parti, des lois sur mesure - produise, aux fins de satire et dénonciation, les objets les plus difformes. Cela advient, en 2003, dans L'anomalo bicefalo (L'Anormal bicéphale), une comédie inscrivant en abyme au creux de son intrigue première le tournage et l'histoire narrée d'un télé-film, ce qui, certes, complexifie la stratification de la "référence" mais connote en permanence la domination médiatique du pouvoir berlusconien : à la suite d'une attaque armée où sont blessés Poutine et Berlusconi, Poutine meurt, et la moitié intacte de son cerveau est greffée sur la moitié intacte du cerveau de Berlusconi ; la vie politique italienne se trouve donc totalement perturbée par cette modification. Dario Fo reprend, pour incarner les formes diverses de perversion du pouvoir, la figure du pupazzo (le pantin) utilisée dès la fondation de la compagnie "Associazione Nuova Scena" en $1968^{30}$. Puisqu'il s'agit ici de fustiger la versatilité politique des communistes et la

29 Le terme d' " anomalie » pour désigner le conflit des intérêts privés du Président du Conseil italien au regard de sa fonction nationale et l'utilisation de son pouvoir législatif pour échapper à toutes ses mises en cause judiciaires a été proposé par des historiens. Cf. Andrea, Di Michele, Storia dell'Italia repubblicana (1948-2008), Milan, Garzanti.

$30 \quad$ En 1968, dans la Grande pantomima con bandiere e pupazzi piccoli e medi (Grande pantomime avec drapeaux et pantins petits et moyens), tandis qu'un dragon (spectre classique de Satan depuis l'Apocalypse de saint Jean et, dans la bande dessinée classique, incarnation du Mal absolu et donc du bolchévisme) tente de s'emparer du pouvoir, l'estomac d'un gigantesque pantin mu par de longues perches crache tous les symboles de la bourgeoisie. L'allusion historique porte, à l'époque, sur les événements de Tchécoslovaquie. 
coupable inaction institutionnelle de la gauche contre Berlusconi pendant les cinq années de son gouvernement (elle n'a fait voter aucune loi pour réprimer le conflit d'intérêts), c'est la danse d'un pantin inanimé et mou - il a le visage de Massimo D’Alema, membre du Parti Communiste Italien puis du Parti Démocratique puis des Démocrates de Gauche puis de la fédération de l'Olivier - avec l'acteur (Dario Fo lui-même, jouant dans la pièce les deux rôles du metteur en scène et de Berlusconi) qui, au rythme endiablé d'un song, assurément moins didascalique et plus intégré à l'action scénique que chez Brecht, signe l'abandon de la lutte de classe et la défaite des idéaux de justice :

Le tango fourbe du compromis.

Un saut par ci

Un saut par là

La lutte de classe on n'en est plus

$[\ldots]$

Faisons-nous baiser, on jouit mieux

Faisons-nous baiser, on jouit mieux

Sautons par là

Sautons en l'air

D'Alema de caoutchouc

Fais-nous danser

Le tango fourbe du compromis. $[\ldots]^{31}$

31 Dario, Fo et Franca, Rame, L'anomalo bicefalo, in Tutto il teatro, Milan, Fabbri Editori, p. 39 : «Il tango furbo del compromesso. / Un salto di qua / Un salto di la' / La lotta di classe piu' non ci sta / [...] Facciamoci fottere, si gode di piu' / Facciamoci fottere, si gode di piu' / Saltiamo di qua / Saltiamo all'insu' / D’Alema di gomma / Facci danzare / Il tango furbo del compromesso. [...] » 


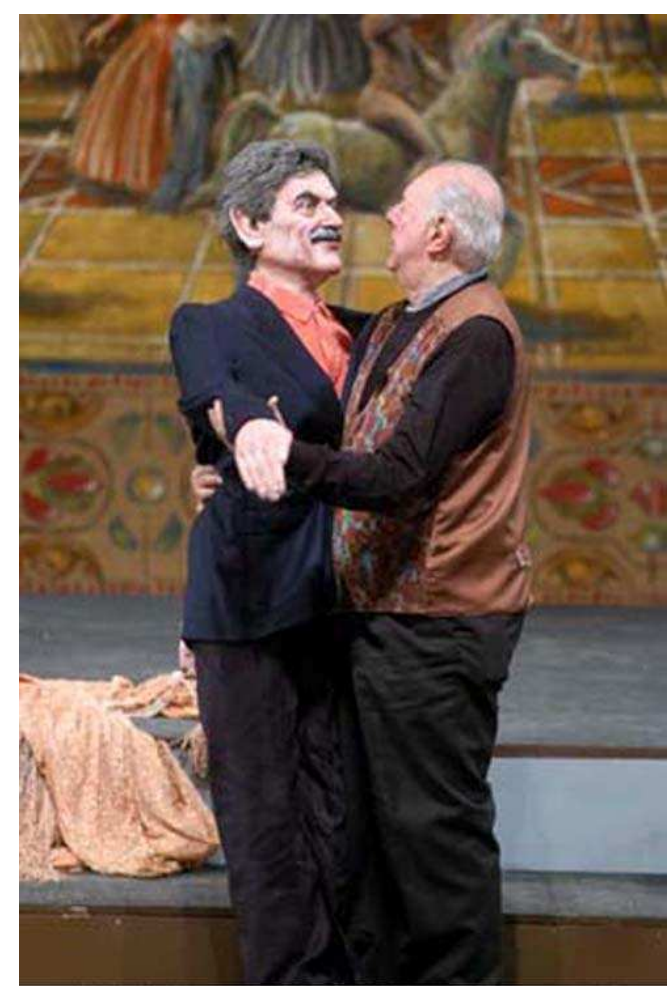

foto di Corrado Maria Falsini - Roma - Italia opera@falsini.com

\section{(C) /archivioforame/ANBI/2004/FOTO/027507/027507-007m}

La dénonciation politique peut être aussi portée par la disproportion affectant le corps humain (dans le personnage de Silvio, il y a tout à la fois redoublement et diminution), et par le décalage généralisé de la fonction des objets attachés à cet humain. Une longue didascalie prévoit la visualisation de la petitesse physique, intellectuelle et surtout morale de Silvio Berlusconi, une sorte de nain, quand le metteur en scène - Silvio s'adresse à l'actrice Anastasia - Veronica (Veronica Lario, épouse de Berlusconi) :

Metteur en scène - Silvio Je ne sais pas... je me sens très confus... (Il se déplace de quelques pas et s'approche de Veronica: ce n'est qu'à présent que nous voyons Silvio dans toute sa «petitesse »: un petit nain. Pour obtenir ce trucage, type Commedia dell'arte, la scène doit être traversée par une fosse, une tranchée de $70 \mathrm{~cm}$ de profondeur où il faut installer l'acteur avec, derrière lui, un mime. L'acteur dépasse de la tranchée de la taille jusqu'à la tête. Ses bras sont enfilés dans un mini pantalon, ses mains sont chaussées d'une paire de chaussures. L'acteur porte un court veston croisé dans les manches duquel sont enfilés les bras du mime. En bref, nous aurons un minuscule nain dont les jambes seront actionnées par les bras et les mains de l'acteur; les bras en revanche seront ceux du mime) $)^{32}$.

32 Ibid., p. 26 : «REGISTA-SILvio Non so... ho un sacco di confusione... (Si sposta di qualche passo avvicinandosi a Veronica : solo ora vediamo Silvio in tutta la sua "bassezza" : un nanetto. Per ottenere questo trucco da commedia dell'arte la scena deve essere attraversata da una 


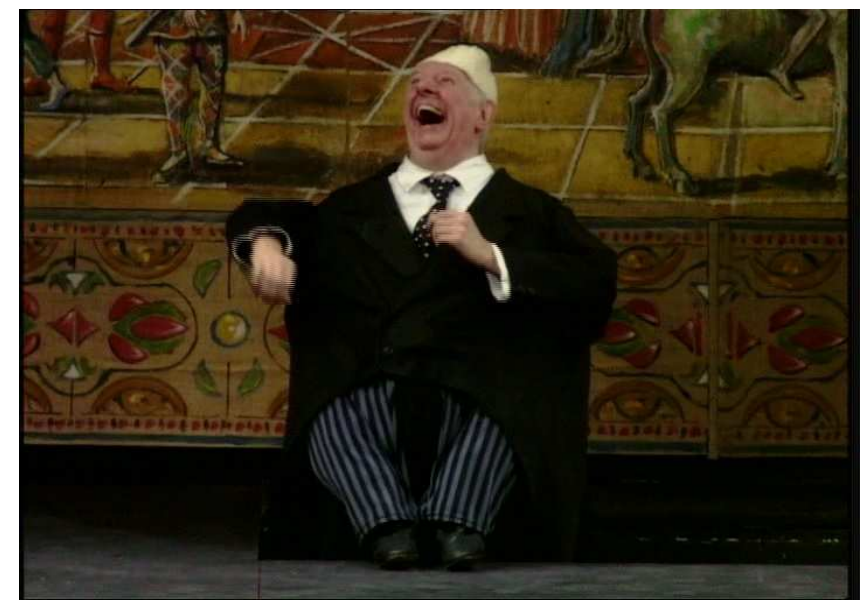

L'anomalo bicefalo, (C) Dario Fo, Franca Rame et Jacopo Fo. Captation à partir de l'enregistrement au Teatro di Varese (21.XII.2003), enregistrement aggiornato le 3 février 2004 au Teatro Bellini de Naples

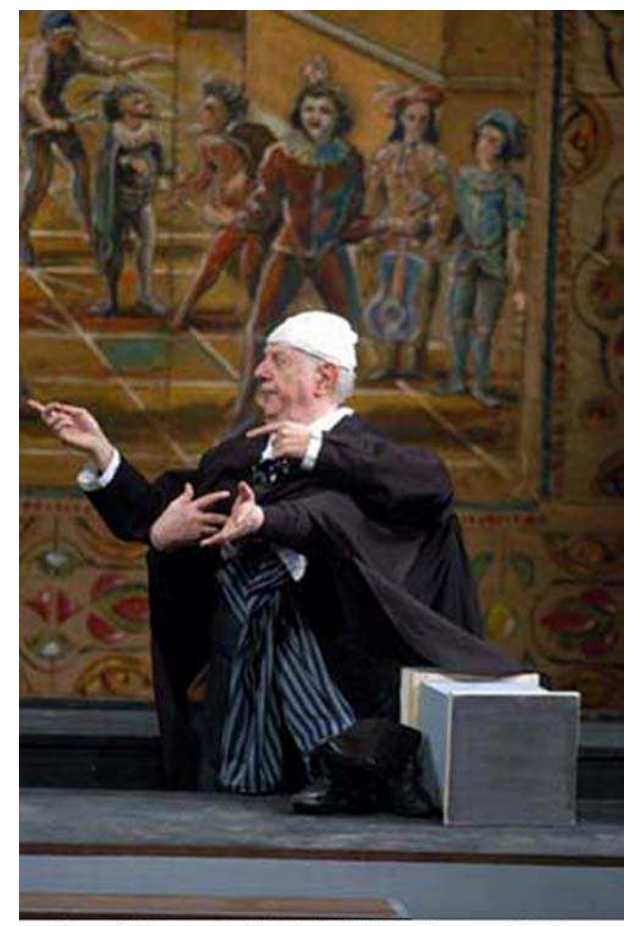

foto di Corrado Maria Falsini - Roma - Italia opera@falsini.com

fossa a trincea profonda $70 \mathrm{~cm}$ dove si va a sistemare l'attore con alle spalle un mimo. L'attore fuoriesce dalla trincea dal bacino in su. Le sue braccia sono infilate in un paio di minipantaloni, le mani calzano un paio di scarpe, L'attore veste un corto doppiopetto scuro nelle cui maniche sono infilate le braccia del mimo. In poche parole, avremo un nanerottolo le cui gambe sono agite dalle braccia e mani dell'attore, le braccia invece saranno prestate dal mimo) » 


\section{(O)/archivioforame/ANBI/2004/FOTO, 027507/027507-003m}

Ce nain, qui ne cesse de tomber du petit cube sur lequel il tente de s'asseoir, souhaitant faire raconter par son épouse le temps heureux de ses débuts matrimoniaux et de ses succès en affaires, entend sur scène raconter les flux financiers de ses sociétés off-shore, l'accumulation des enquêtes auxquelles il a été soumis et l'exposé de ses amitiés mafieuses. Mais la description échappe à toute platitude mimétique qui arrêterait la réception dans la dénotation, parce que l'écoute du spectateur se heurte au choc visuel que constitue cette difformité du corps humain et de son rapport aux objets quotidiens mis en scène : au-delà du rire qu'il déclenche, le grotesque alerte le spectateur, ouvre son cerveau. Alors, naît l'indignation face au comportement réel de Berlusconi dans la société italienne.

\section{Un objet de lutte}

L'objet que mime le "jongleur" ou que manipule l'acteur est certes intégré à un travail scénique parfaitement maîtrisé qui affirme la vitalité d'un thêâtre "populaire" et "total" dépassant toutes les frontières génériques et inter-arts. Mais, parce que le théâtre n'a pas vocation à maintenir sa force dans les étroites limites de la scène, parce que, selon Dario Fo, il est une tribune et le lieu de fondation d'une communauté de spectateurs, une forme moderne de l'agora, il doit engager le spectateur à être, dans l'urgence politique italienne et internationale, présent et actif pour redonner place à la démocratie. L'objet scénique devient ainsi une arme dans la Cité.

\section{Bibliographie}

\section{Textes}

FO, Dario, Mystère bouffe. Jonglerie populaire, traduit et adapté par Ginette Herry, Paris, Dramaturgie Éditions, 1984.

FO, Dario, Dialogo provocatorio sul comico, il tragico, la follia e la ragione con Luigi Allegri, Bari, Laterza, 1990.

FO, Dario, Le Gai Savoir de l'acteur, traduction française de Valeria Tasca, Paris, L'Arche, 1990.

FO, Dario, Teatro, Franca Rame éd., Turin, Einaudi, 2000.

FO, Dario et RAME, Franca, L'anomalo bicefalo, in Tutto il teatro, Milan, Fabbri Editori, 2006.

FO, Dario, Manuale minimo dell'attore, Turin, Einaudi, 2001 (1ère éd. 1987).

MOLIĖRE / DARIO FO, Le médecin malgré lui et Le médecin volant, collection «Le spectateur français » dirigée par Jean-Loup Rivière, Imprimerie nationale Éditions, 1991.

Site internet www.archivio.francarame.it

\section{Ouvrages critiques}

DELEUZE, Gilles, L'Image-mouvement, Paris, Les Éditions de Minuit, 1983. 
DI MICHELE, Andrea , Storia dell'Italia repubblicana (1948-2008), Milan, Garzanti, 2008.

La Repubblica, 10 octobre 1997, p. 15 section Culture.

MÉLIÈS, Georges, "Les vues cinématographiques », in Annuaire général et international de la Photographie, Paris, Plon, 1907 ; à présent in SADOUL, Georges, Georges Méliès, Paris, Seghers (Cinéma d'aujourd'hui).

PAVIS, Patrice, Dictionnaire du théâtre, Paris, Éditions sociales, 1980.

QUADRI, Franco, L'avanguardia teatrale in Italia (II. Materiali 1960-1976), Turin, Einaudi, 1977.

UBERSFELD, Anne, «L'objet thêâtral », in Actualité des arts plastiques, $\mathrm{n}^{\circ}$ 40, Paris, CNDP, 1978 ; «L'objet », in Les termes clés de l'analyse du théâtre, Paris, Seuil, 1996.

\section{Pour citer le document}

Dominique Budor, «Détournement et invention de l'objet scénique dans le thêâtre de Dario Fo», Agôn [En ligne], Dossiers, N4 : L'objet, Objet, objection, objecteurs, mis à jour le : 25/01/2012, URL : http://agon.ens-lyon.fr/index.php?id=2056 